\title{
Health system governance to support scale up of mental health care in Ethiopia: a qualitative study
}

\author{
Charlotte Hanlon ${ }^{1,2^{*}}$ (D) Tigist Eshetu', Daniel Alemayehu', Abebaw Fekadu ${ }^{1,3}$, Maya Semrau², \\ Graham Thornicroft ${ }^{2}$, Fred Kigozi ${ }^{4}$, Debra Leigh Marais ${ }^{5}$, Inge Petersen ${ }^{6,7}$ and Atalay Alem ${ }^{1}$
}

\begin{abstract}
Background: Ethiopia is embarking upon a ground-breaking plan to address the high levels of unmet need for mental health care by scaling up mental health care integrated within primary care. Health system governance is expected to impact critically upon the success or otherwise of this important initiative. The objective of the study was to explore the barriers, facilitators and potential strategies to promote good health system governance in relation to scale-up of mental health care in Ethiopia.
\end{abstract}

Methods: A qualitative study was conducted using in-depth interviews. Key informants were selected purposively from national and regional level policy-makers, planners and service developers $(n=7)$ and district health office administrators and facility heads $(n=10)$ from a district in southern Ethiopia where a demonstration project to integrate mental health into primary care is underway. Topic guide development and analysis of transcripts were guided by an established framework for assessing health system governance, adapted for the Ethiopian context.

Results: From the perspective of respondents, particular strengths of health system governance in Ethiopia included the presence of high level government support, the existence of a National Mental Health Strategy and the focus on integration of mental health care into primary care to improve the responsiveness of the health system. However, both national and district level respondents expressed concerns about low baseline awareness about mental health care planning, the presence of stigmatising attitudes, the level of transparency about planning decisions, limited leadership for mental health, lack of co-ordination of mental health planning, unreliable supplies of medication, inadequate health management information system indicators for monitoring implementation, unsustainable models for specialist mental health professional involvement in supervision and mentoring of primary care staff, lack of community mobilisation for mental health and low levels of empowerment and knowledge undermining meaningful involvement of stakeholders in local mental health care planning.

Conclusions: To support scale-up of mental health care in Ethiopia, there is a critical need to strengthen leadership and co-ordination at the national, regional, zonal and district levels, expand indicators for routine monitoring of mental healthcare, promote service user involvement and address widespread stigma and low mental health awareness.

Keywords: Governance, Systems thinking, Health systems, Developing country, Mental health services, Mental health policy, Primary care

\footnotetext{
*Correspondence: charlotte.hanlon@kcl.ac.uk

${ }^{1}$ Department of Psychiatry, School of Medicine, College of Health

Sciences, Addis Ababa University, Addis Ababa, Ethiopia

Full list of author information is available at the end of the article
} 


\section{Background}

Integration of mental health into primary care services is the core recommendation of the World Health Organization (WHO) mental health Gap Action Programme (mhGAP) [1] in order to improve access to evidencebased mental health care in low- and middle-income countries (LMICs). However, although evidence is accruing for the efficacy of specific treatment packages for prioritized mental, neurological and substance use disorders in LMIC settings, the evidence base to support realworld effectiveness of implementation at scale is lacking [2].

\section{Systems thinking and health system governance}

Expert consensus and the experience of those seeking to implement integrated primary mental health care indicate that health system factors are likely to be critical [3, 4], in keeping with lessons learned from roll out of other global health programmes [5]. The WHO has delineated the following interlinked 'building blocks' of the health system: service delivery, health workforce, information, medical products and technologies, financing and governance [6, 7]. Systematic evaluation is needed of the impact of health system components and processes on mental health care scale-up, with attention paid to the dynamic interactions between system components and their interface with the wider political and organisational context [7]. Strategies can then be developed to strengthen mental health systems across varied LMIC settings. Such 'systems thinking' approaches are in their infancy in the field of global mental health [8], particularly in relation to governance. Health system governance (HSG) has been defined by WHO as "ensuring that strategic policy frameworks exist and are combined with effective oversight, coalition building, regulation, attention to system-design and accountability" [6]. Good health system governance has been conceptualised as a fundamental requirement for optimal functioning of all other health system components [9] and, therefore, forms a critical focus of enquiry.

\section{Mental health context in Ethiopia}

Ethiopia is a low-income country in sub-Saharan Africa with a population approaching 100 million people [10]. Specialist mental health professionals are scarce. There are approximately 60 psychiatrists practicing in the public sector, with most located in the capital city of Addis Ababa, although psychiatrist-led services have recently expanded to regional centres [11]. Masters level psychiatric practitioners and Bachelors degree level psychiatric nurses provide hospital-based mental health care across the country, but these services are limited to urban centres. As a consequence, the treatment gap for mental health care is very high, with an estimated $90 \%$ of people with severe mental illnesses (including schizophrenia and bipolar disorder) never receiving evidence-based care, and less than $1 \%$ receiving continuing care [12]. Rigorous epidemiological studies have shown that the burden of mental health problems in Ethiopia is high $[12,13]$, with untreated mental health conditions associated with premature mortality [14], disability [15], adverse economic impacts [16], stigma, discrimination and human rights abuses [17, 18]. In response, the Federal Ministry of Health in Ethiopia is embarking upon a ground-breaking programme to scale up access to mental health care using the WHO model of integration into primary care.

\section{Policy and health system context}

Governance of the Ethiopian health system operates at multiple levels [19]. The Federal Ministry of Health provides central direction with the national level health policy and five yearly health care plans which integrate all health conditions [20]. Each of Ethiopia's nine regions and the city administrations can exercise some autonomy in terms of implementation of health care plans, although are constrained to meet nationally set targets for health care delivery and health outcomes. At the lowest level of health care planning within the system, District Health Offices are responsible for supplying a range of services tailored to the specific needs of the population that they serve and are expected to lead health care planning from the grassroots, through 'district-led planning.'

In the current five year plan, there is a target to expand mental health care to $100 \%$ of districts by 2020 and to increase the number and range of routinely collected health management information system indicators for mental health [19]. The National Mental Health Strategy of Ethiopia provides a more detailed framework for integration of mental health into the primary care system [21]. Ethiopia has no legislation concerning the provision of treatment to people with mental illness against their will and there is no specific legislation to protect the rights of people with mental health conditions in the wider society [22]. However, Ethiopia is a signatory to the United Nations Convention of the Rights of People with Disability which includes the rights of people with mental disability [23]. New social and community-based insurance initiatives aim to reduce the catastrophic costs associated with out-of-pocket expenditure for health care [24].

In collaboration with the WHO, mhGAP was piloted in Ethiopia across four regions [25], leading to a governmental commitment to scale up mental health care through in-service training of health centre-based primary care workers using mhGAP intervention guidelines [26] and integrating mental health into the packages of care 
delivered by community-based health extension workers [27]. Research-led programmes to implement and evaluate integrated mental health care are also underway in Ethiopia, including the Programme for Improving Mental health carE (PRIME) [28, 29], the Africa Focus on Intervention Research for Mental Health (AFFIRM) [30] and the 'Emerging mental health systems in LMICs' (Emerald) programme [31].

The goal of the Emerald programme is to generate evidence and capacity to strengthen health systems to scale up mental health care and thereby improve mental health outcomes in six LMICs: Ethiopia, India, Nepal, Nigeria, South Africa and Uganda [31]. Emerald focuses specifically on the health system functions of financing, service delivery, information and governance. Qualitative studies have been conducted in all Emerald countries to explore HSG in relation to the mental health system, with published findings available from Nigeria [32], South Africa [33] and Uganda [34]. In this paper we report findings from the qualitative study conducted in Ethiopia in order to address the following research questions:

- What are the institutional, contextual and health system governance barriers and facilitators to scaling up mental health care in the Ethiopian setting?

- What system level strategies may be employed to maximise the success of scale-up in Ethiopia?

\section{Methods}

\section{Study design}

A qualitative study was conducted using the HSG evaluation framework developed by Siddiqi and colleagues [35], adapted to include the inter-relationships with other health system components [9] and for the specific case of mental health care integration into primary care [33].

\section{Setting}

Ethiopia is a low-income country located in the Horn of Africa, ranked 174th out of 188 countries in the Human Development Index [36]. As recommended [35], the evaluation of HSG was conducted at both the national/ regional level and at the district level. The PRIME implementation site was selected as the district site because plans were being developed there to integrate mental health care into primary care $[28,29]$. PRIME is operational in Sodo district of the Gurage Zone, Southern Nations, Nationalities and Peoples Region, located around $100 \mathrm{~km}$ from the capital city of Addis Ababa. The district has a population of approximately 160,000 people and is almost $90 \%$ rural and predominantly Orthodox Christian in religion [28]. At the time of the study there were eight primary care health centres (one operating as a public-private partnership), around 54 frontline health posts staffed by community health extension workers and no mental health services in the district.

\section{Sample}

Key informants were selected purposively from the national/regional and district level. At the national/ regional level, planners $(\mathrm{n}=3)$ and mental health leaders involved in service development $(\mathrm{n}=4)$ participated. At the district level, key informants were sought from planners $(\mathrm{n}=2)$ and health facility managers $(\mathrm{n}=8)$ located in Sodo district. Sampling at the national and regional level was constrained by the small number of people involved in mental health care policy-making and planning. Similarly at the district level, sampling was limited by the availability of managers and planners who had exposure to the concept of integration of mental healthcare into primary care. All those who were invited to participate accepted the invitation.

\section{Data collection}

Semi-structured interviews were carried out using a topic guide derived from the adapted HSG evaluation framework [33]. See Additional file 1. This HSG framework seeks to ensure comprehensive coverage of all aspects of health system governance. The main domains covered by the topic guide were: strategic vision and rule of law, collaboration and participation, responsiveness and integration, equity and inclusiveness, effectiveness and efficiency, information, transparency and accountability, and ethics, considered in relation to health system financing, human resources, medication and technologies, information systems, infrastructure and service delivery.

The national level informant interviews were conducted face-to-face in English by $\mathrm{CH}$ and AA, apart from two interviews with regional participants that were conducted by email due to time constraints and geographical inaccessibility of the informants. The district level interviews were conducted in Amharic, the official language of Ethiopia, by Masters level research assistants who were trained in qualitative methods. The interviews were transcribed in Amharic and then translated into English, with discussion in the Ethiopia team to resolve uncertainties over translation.

\section{Data analysis}

A framework approach to analysis was taken [37]. An initial coding framework prepared for cross-country application was adapted at baseline for contextual relevance and modified further as coding progressed. The framework is presented in Additional file 2. The Siddiqi framework provided the parent themes, but sub-themes and child themes were derived from the data and reflected the Ethiopian context for scale-up. Coding of transcripts was 
carried out by $\mathrm{CH}, \mathrm{TE}$ and DA. The coding team met frequently to allow inductive adaptation of the coding framework, to clarify the scope of specific codes and to ensure consistent application of codes to the data. Open source software (Opencode 4.0 [38]) was used to facilitate the handling and analysis of the qualitative dataset. Once coding was complete, TE and DA extracted the coded data and summarised the findings by participant and by code/ theme in a matrix. The participants were grouped according to type of informant. The data summaries were then compared across participants and participant groups.

The preliminary findings from the study were presented at a meeting, which was convened by the Federal Ministry of Health in collaboration with Emerald, in July 2015 in order to explore the system barriers to scale-up of mental health care in Ethiopia. Participants included the mental health focal persons and representatives from Regional Health Bureaus for the regions involved in scale up of mental health care in Ethiopia.

\section{Ethical considerations}

Ethical approval was obtained from the Institutional Review Board of the College of Health Sciences, Addis Ababa University (Reference No. 074/13/Psy). Participation was voluntary and only took place after informed consent had been given. Care was taken in the presentation of quotations to ensure that respondents could not be identified.

\section{Results}

The findings are presented in relation to the adapted framework for analysing HSG [33], stratified by national/ regional and district level respondents where differences were apparent. A summary of the findings is presented in Table 1. Further supporting data for each theme are included in Additional file 3. The matrices used for analysis of the district level informants are included in Additional files 4 and 5 .

\section{Strategic vision and rule of law}

Almost all national/regional level respondents spoke about the positive government support for improving access to mental health care in Ethiopia.

"I really think that there is a certain momentum around mental health in Ethiopia, I can see that.

I'm not aware of other countries in this region that have made such a progress" (National level, ID6).

Particular emphasis was given to the high levels of governmental support at the national level. The value of the National Mental Health Strategy in solidifying this support in the longer term was seen as vital. A district level respondent considered the Strategy essential to give districts a mandate to expand mental health care. The assignment of a mental health focal person to be situated within the non-communicable diseases (NCDs) section of the $\mathrm{FMOH}$ was seen as a positive development for some but an issue of concern for others. On the positive side, NCDs were considered to be a higher political priority than mental health so that mental health would benefit from the close linkage. On the other hand, some respondents were concerned that other NCDs would be prioritized over mental health. The financial commitment from the Ministry of Health to mental health care scale-up was viewed by most national level respondents as important evidence of governmental support and a strong facilitator to the success and sustainability of scale-up.

Most respondents at the national level endorsed the desirability of mental health legislation, but were divided on the extent to which it was a current priority or, indeed, a necessary prerequisite to scaling up mental health care. The need for legislation that was workable within the Ethiopia context was emphasised rather than importing Western models.

\section{Planning and co-ordination}

Most national level respondents reported that there was little expertise for mental health care planning at all administrative levels in the health system (including regional health bureaus and district health offices) because of the newness of the programme and the low baseline level of mental health care provision. As a consequence, there was little experience in mental health care planning and it was difficult to predict demand for services. There was optimism amongst some respondents that most of these issues would improve as the scale-up progressed.

In view of the decentralized system of health care planning in Ethiopia, all respondents observed that nationallevel policies could only be implemented successfully with the 'buy-in' of the Regional Health Bureaus, which was currently lacking due to low awareness.

"The district and zonal health office is at best ignorant about mental health services. Mental health is totally out of the planning and management" (National level, ID2).

The experience gained to date had underlined the need for committed mental health care co-ordinators at each organisational level.

\footnotetext{
"We were trying to use ... persons who were assigned as focal persons at the Regional Health Bureau to help to coordinate activities in the health facilities ... But these people have other duties... Somebody who can play that role would be very critical I think because this programme will improve or go down, depending on its success..." (National level, ID7).
} 


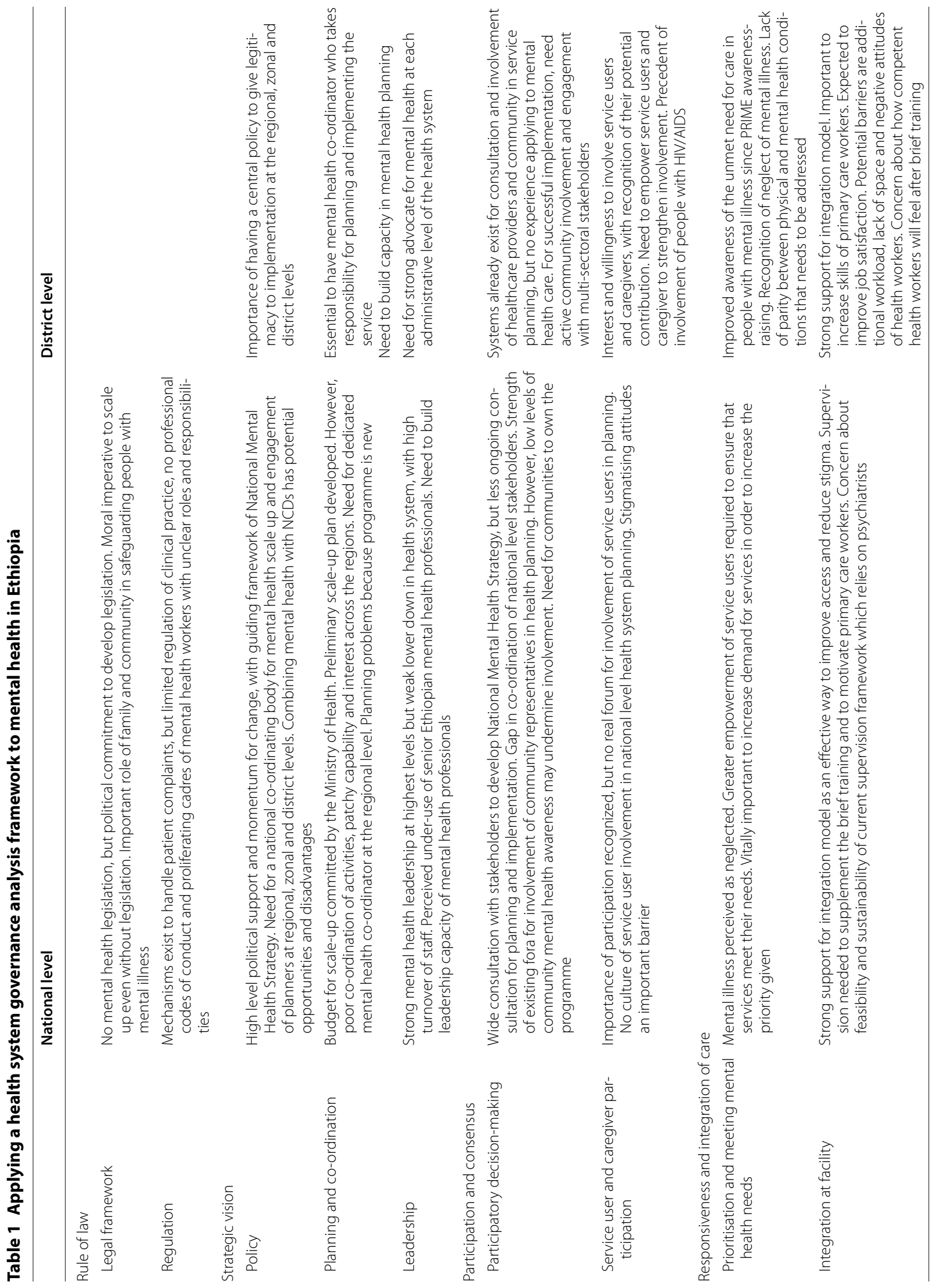




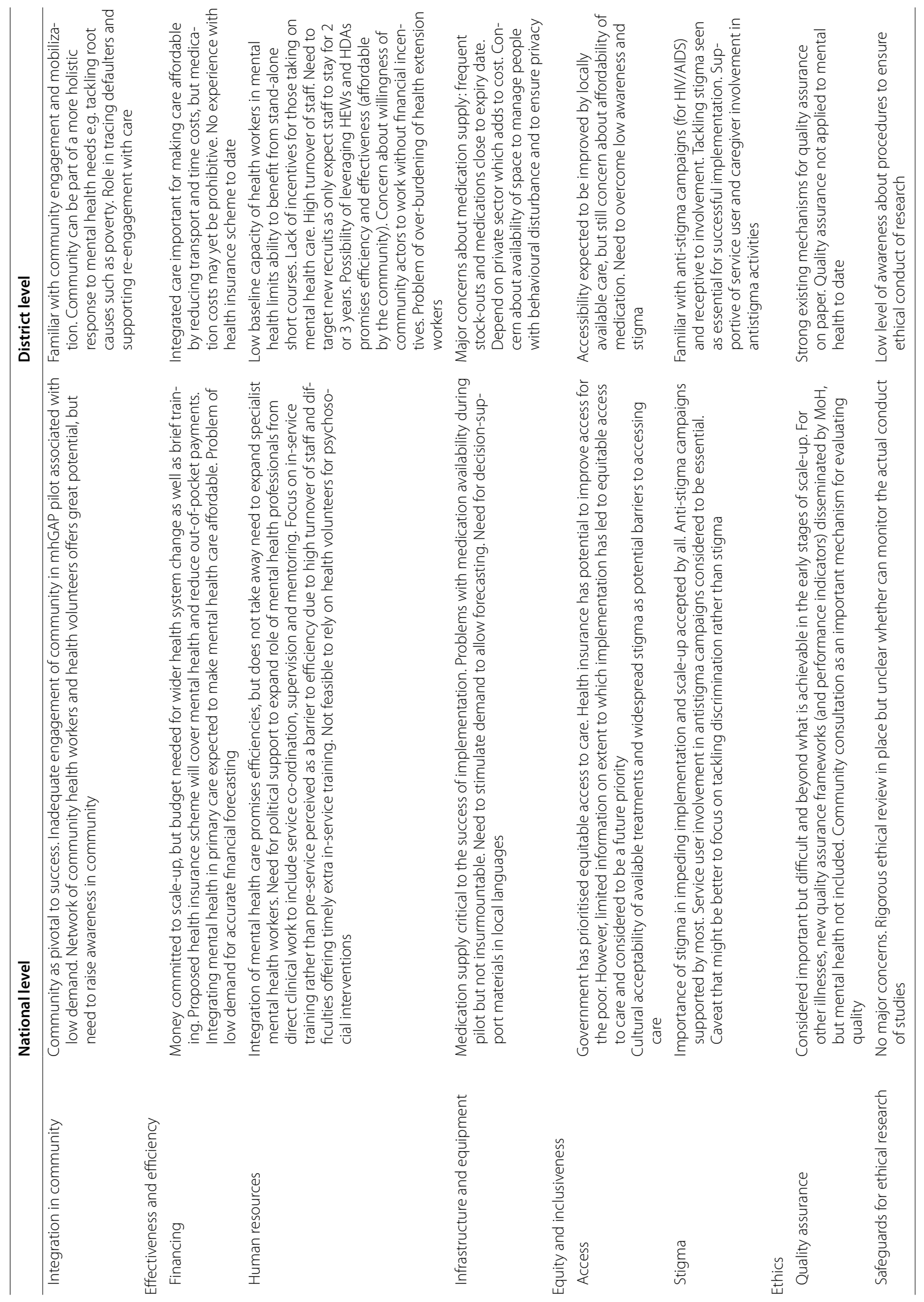




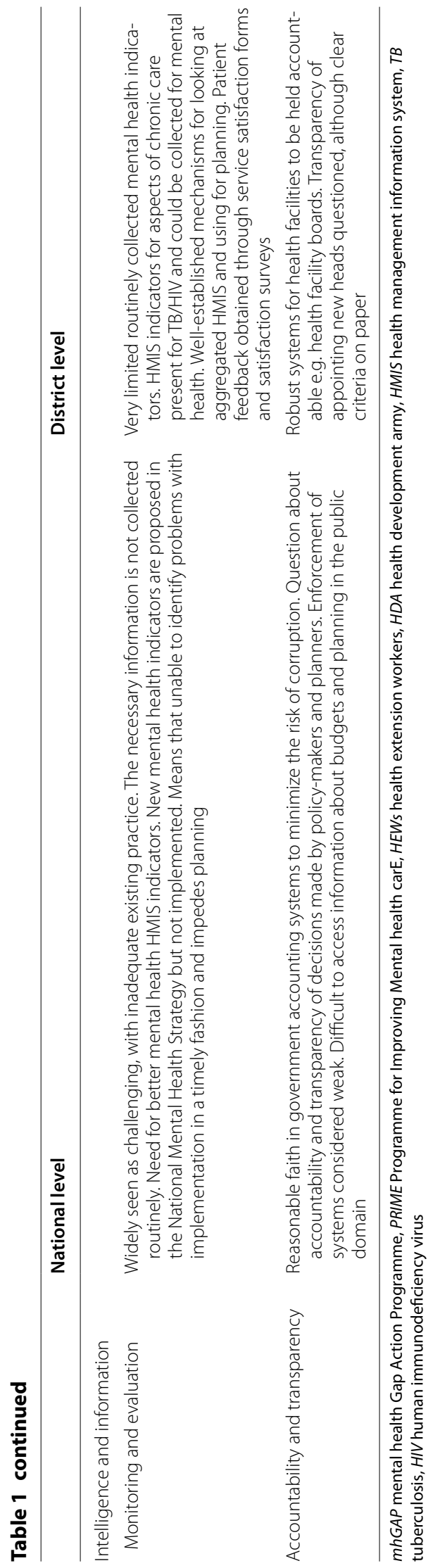


A co-ordinator would be able to ensure adequate budget allocation and reliable supplies of medication, to oversee the monitoring and evaluation activities and to lead awareness-raising in the community. Several district level respondents also spoke of the need for clear directives flowing from the Federal Ministry of Health downwards.

Some respondents felt that mental health professionals were best placed to lead service planning and co-ordination, but that this approach was limited by both the small numbers of specialists and their lack of training and experience in leadership roles. The need to strengthen mental health leadership among non-mental health professionals currently involved in the general planning of healthcare was also thought to be essential. Respondents reported a range of barriers to achieving this goal: the high turnover of staff in these positions, the difficulty in recruiting appropriately skilled individuals due to low salaries, the lack of interest in mental health issues and stigmatizing attitudes towards mental health.

\section{Participation and consensus}

The process of development of the National Mental Health Strategy was endorsed as participatory by most national level respondents. However, this spirit of consultation and participation was reported to have been less prominent following the launch of the Strategy and during the shift to the next phase of national planning and implementation. In particular most respondents perceived there to be a lack of involvement of the community, service users and non-health sectors in the planning of mental health scale-up.

"Decisions are usually made from top down, with very little opportunity for participatory approach" (National level, ID3).

An important barrier to greater involvement of stakeholders in the planning process was the absence of any formalised structure to facilitate involvement, for example, in the form of a committee or designated body.

District level respondents had little experience with mental health care planning but highlighted the existing mechanisms to ensure participatory planning for health services more generally. One respondent spoke of the value of involvement of key stakeholders from outside of the health system in the planning process, for example the police or organisations involved in promoting livelihoods, so that they would then be more active in their support of implementation and allow multi-sectoral coordination. Community participation in planning new services was seen by almost all district level respondents as vital to the success of the endeavour.
"People at the grass root level should be convinced on the prepared plan otherwise we cannot achieve our goal" (District level, ID10).

When asked directly, most district level respondents appeared receptive to the involvement of service users and caregivers in service development, although not necessarily in the initial planning of the new mental health service. They drew from their experiences of working with people with HIV to develop and improve services but acknowledged that they had no experience of working collaboratively with people with mental health problems.

\section{Responsiveness and integration Prioritisation and meeting mental health needs}

At the national level, all respondents recognised a high unmet need for mental health care and none doubted the need to expand mental health care. However, some respondents anticipated that there would be low levels of awareness within the regional, zonal and district health administrations about the unmet mental health needs of their populations. Several respondents identified a need to improve the capacity of Regional Health Bureaus to identify the specific mental health needs of their populations and plan accordingly. The existing system of periodic client satisfaction surveys was identified as one approach to identifying unmet needs. Most respondents expressed confidence that planners would respond to increased demand for services.

District level respondents spoke similarly of the neglect of mental health care at every level of the system. They reported that their recognition of the unmet needs of people with mental illness had been improved through the awareness-raising carried out by the PRIME demonstration project. Low awareness and stigmatising attitudes were identified by all district level respondents as reasons for the low priority given to mental health care.

"I think it is good, as we know mental health and people with this problem have been ignored. They have been suffering a lot and also didn't get a proper care, and they have been referred to Addis Ababa and to other far places instead of getting treatment easily and get better like the other patients. In addition to this the society have negative attitude towards them..." (District level, ID17).

\section{Integration at facility level}

There was strong support from almost all national level respondents for the plan to integrate mental health into primary care services. A potential barrier identified by a 
few respondents was the availability of adequate supervision for newly trained primary care workers.

"I think based on the programme we are running, we cannot do it without supervision and mentoring.... So the training is short training, and people are just told about different illnesses and so they have to integrate it into their practice. And by mentoring and supervision, we are able to I think fill in that gap and also keep them motivated to work as part of their practice because these general health workers are not used to helping mentally ill people" (National level, ID7).

Current models of centrally-based psychiatrists providing supervision and mentoring to newly trained primary care workers were seen as unsustainable due to the small numbers of available psychiatrists, their competing commitments and the cost of paying for their involvement. Making use of the expanding cadres of non-psychiatrist mental health specialists, for example psychiatric nurses and Masters level psychiatric practitioners, was raised as a workable alternative. However, there was some uncertainty about whether the existing system would be able to support a shift in the role of these mental health professionals, from clinician to that of trainer and supervisor.

"Because currently, mainly their role is to just help patients who come to see them. But now this is an additional role. They'll have to go out of their routine clinical practice to help in the training and supervision or mentoring" (National level, ID7).

Another concern expressed by a national level respondent was about the acceptability of biomedical approaches to mental health care to most people in the community.

"It could be the way, but I am not entirely convinced that people will show up to a health centre just because someone is there to give care; there are more complex issues why people do not access modern mental health care which need addressing" (National level, ID3).

Almost all district level respondents thought that the needs of people with uncomplicated mental illness would be better served by receiving care in the primary care setting due to closer proximity, lower transport costs and lower time burden for patients and the accompanying caregivers.

"I appreciate the idea of integrating mental health service to primary health care. This makes the service easily reachable to the society at large" (District level, ID10).
District level respondents also anticipated that increasing treatment coverage for people with mental health problems would lead to reduced suffering and stigma. Timely intervention was expected to lead to better illness outcomes and to reduce the chance that mental health problems would develop into a more serious disorder.

One facilitating factor for introducing the new service model was a reported interest of primary healthcare workers to expand their areas of clinical competence. One respondent spoke of the enhanced job satisfaction that would result from being trained to deliver mental health care.

\section{"The other benefit is that the health professional will get satisfaction at least by giving a complete service. They also became competent and a better profes- sional" (District level, ID12).}

Despite the positive attitude of most respondents, some respondents expressed concerns about the additional workload that would result from delivery of mental health care and the lack of time to see complex patients in the usual out-patient clinic. A few respondents anticipated that some health workers would be reluctant to get trained and that the additional workload could even reinforce negative attitudes towards people with mental health problems.

\section{Integration in the community}

The success of integrating mental health into primary care was reported by almost all respondents to be dependent on concomitant mobilization of the community. A relative lack of involvement of the community was seen as a limitation of current implementation activities, leading to low demand for mental health care at primary care facilities.

Many of the respondents detailed the focus given in the Ethiopian health system to community engagement and mobilisation in response to health issues. Respondents were quick to link the potential of this existing system to the plans for mental health care integration. Community-based health extension workers were seen as the natural gateway into the community, providing access to a network of health volunteers. Respondents were familiar with using these community structures to address other health conditions, although not mental health. From their experience with TB and HIV, for example, respondents reported that the community network could improve case-finding, raise community awareness, reduce stigma, engage traditional and religious healers and help track loss to follow up. The need to engage the community so that they own the programme was emphasised by all. Respondents also identified a potential role 
of the community-based health network in addressing the restraint of people with mental illness in their homes.

"It is important, for example, they are in the community, they know all people in that sub-district and people who are tied in the households because they always go door to door, so, I think they are appropriate to identify and to create awareness in the society and bring them to the health facility if they can get simple training regarding mental health, so, it will be good if they can get training" (District level, ID17).

Respondents noted that non-health structures within the community had a critical role to play in the successful implementation of integrated mental health care. As well as speaking to the critical role of traditional and faith healers and community leaders, many respondents spoke spontaneously about the role of engaging the nonhealth sector as part of the response to mental ill-health. This was noted both in relation to rehabilitation and to respond adequately to the root causes of disengagement from care and non-adherence to medication, most notably poverty.

"When we come to mental health, the religious institutions have big roles...okay... they are the one(s) who can support us. .... I think we should collaborate with priests, edir [funeral group] leaders, and people who are working in agriculture and education sector." (District level, ID 17).

Respondents identified potential barriers to successful integration of mental health into existing community structures: the variable performance of different sub-districts and the difficulty in making interventions sustainable when relying on volunteers. An additional barrier articulated by one respondent was that health extension workers are known to have expertise in specific areas and that the community may not be convinced by the expansion of their role into mental health. However, other respondents referenced similar concerns about HIV, which had been shown to be surmountable. Some respondents were concerned about the capability of health extension workers and volunteers to grapple with mental health.

\section{Effectiveness and efficiency \\ Financing}

Concern was expressed by a couple of national level respondents that the existing budget allocation focused narrowly on training for primary care workers and may not be sufficient to support the necessary health system changes to support scale-up of mental health care.
However, there was appreciation from one respondent that the government had dedicated money towards scaleup activities.

At the district level, almost all respondents expressed concern about the prohibitive costs of out-of-pocket payments for medication needed on a long-term basis to treat mental illness.

"I don't think a person with mental health problem can afford the price of these medicines. We have to see in Ethiopian context...I think we have to give them freely. Mental health medicines are expensive. If it is given freely it should be supplied sustainably." (District level, ID13).

However, improving accessibility of mental health care through integration into primary care was seen by most as a positive intervention to decrease the financial burden on individuals. The planned roll-out of community-based health insurance and social insurance was welcomed.

\section{Human resources}

Making use of existing non-specialist health personnel to expand the reach of mental health care was seen as an efficient use of resources by most respondents. However, national level respondents were quick to point out that task sharing did not eliminate the need to expand specialist mental health professionals, as specialists would also be needed to ensure successful scale-up. Another concern was the efficiency of in-service training of primary care workers. One major barrier was the high level of turnover of primary care staff in rural areas, who were reported to often only stay in post for 2 or 3 years before moving to urban settings or to positions in non-governmental organisations. The drivers for high turnover were reported to be low salaries and limited opportunities for health workers to supplement their salaries, poor living conditions in rural areas and a lack of access to educational opportunities. To address this challenge, respondents argued for a focus on pre-service training.

"What I would advocate for, which I think can really make a difference is reviewing the curriculum and having good integration of mental health in the curriculum because that saves even more money" (National level, ID5).

Respondents spoke of some limitations in the ability of health facilities and district administrators to plan for recruitment and training of staff. Provision of inservice training in mental health care was reported to be dependent on external resources being made available and could not, therefore, be scheduled to meet the need as and when it arose. 


\section{Infrastructure and medication}

The provision of reliable supplies of psychotropic medication was the main concern for most respondents. Numerous potential barriers were identified by respondents. Procurement systems for all medications were reported to be centralised, bureaucratic and lengthy. As a consequence, respondents reported stock-outs and supply of medications that were very close to their expiry date, obliging the primary healthcare facilities to purchase medications from private suppliers at a much higher cost. The difficulty in predicting demand compounded the problem.

District level respondents expressed concern about the lack of appropriate space for assessing and treating a person with mental illness. A particular concern was that people with acute behavioural disturbance would disrupt the clinic or block the clinic because they required a longer period for their consultation.

"... it needs a well organised department, it shouldn't be mixed with other types of services, and I don't think there are health centers which have a separate room for mental health care service; but it will be good if we can have a quiet and confidential places for mental health patients" (District level, ID15).

Respondents endorsed the need for up-to-date materials, for example treatment guidelines, to support the delivery of mental health care. It was suggested that such decision support materials would be more effective if they were translated into local languages.

\section{Equity and inclusiveness}

\section{Access to services}

Government policies were reported to promote equitable access to all types of care, for example through the planned health insurance scheme, as well as broader policies to reduce poverty and increase education. Nonetheless, national respondents noted that there was a lack of information about the extent to which integrated mental health care was in fact accessed by vulnerable segments of society. One national level respondent acknowledged the importance of equity but saw this as something to be addressed later down the line, once the scale-up was underway.

\section{Stigma}

At the national level, stigma was recognised as a potent barrier to the successful scale-up of mental health care. Most respondents supported the idea of anti-stigma campaigns in an attempt to reduce stigma and had either participated in such events or expressed willingness to do so. District level respondents were clear that low levels of awareness and the presence of stigmatising attitudes were common in the community and a potential barrier for people with mental illness to access mental health care. The notion of community awareness-raising and anti-stigma campaigns was familiar to the district level health centre heads and administrators from their experiences with HIV/AIDS, although none of the respondents had been involved in mental health-related activities. All respondents expressed willingness to be involved and considered this to be part of the role of a primary care worker and necessary for the success of mental healthcare scale-up. Respondents valued the idea of involving people with mental illness who had recovered or their caregivers in awareness-raising activities and expressed the view that this would increase the impact on the community.

"We can take the HIV anti-stigma as an example. We were successful because the patients expose themselves and teach the society share their experience. So patients and caregivers should participate on this anti stigma campaign" (District level, ID12).

\section{Ethics and quality assurance}

National level respondents acknowledged the importance of quality assurance but mostly considered this to be beyond what was achievable at this early stage in the implementation and scale-up of mental health care. The existence of quality assurance frameworks and performance indicators, disseminated by the Ministry of Health, was seen by most as a facilitating factor for future quality assurance in mental health care. Even though none of the quality assurance measures had been applied to mental health, some respondents were confident that this could be the case. Many district respondents spoke of the importance of getting feedback from the community in order to obtain a true sense of the adequacy of care.

\section{Intelligence and information}

Deficits in the monitoring and evaluation of the scaleup of mental health care were seen as a critical barrier to scale-up by most national level respondents. Parallel systems of data collection were required because of the lack of mental health indicators included within the routine health surveillance systems. As a consequence it was difficult to obtain information about the functioning of the system and to detect problems in a timely fashion.

"The information being gathered based on the routine information and data collection by the Ministry of Health does not help very much to get the right information... So I think the indicators that are useful, too, in helping the different level decision-makers are not available" (National level, ID7). 
Prioritisation of this area by the government was noted, through inclusion of new indicators in the National Mental health Strategy, although these had not been implemented. Respondents in the district spoke of the usefulness of the existing systems for monitoring and evaluation, covering every level within the health system.

\begin{abstract}
"The advantage of giving feedback is, (that) it will indicate activities which are not performed; for example the district administration can comment to the health centres about activities which haven't be(en) done based on the data at hand. For example, there might be many cases in one administrative sub-district but they are working on some of them only, but if there are such type of processes, they will be forced or obligated to find the remaining cases and bring them to the health facilities" (District level, ID8).
\end{abstract}

\section{Accountability and transparency}

The existence of systems to ensure accountability was reported by most respondents, although some questioned the adequacy of their implementation. However, at the district level, many of the respondents felt themselves to be accountable to the community for planning decisions and service quality via regular public discussions and the feedback of the health boards affiliated to each health facility. Similarly the systems to ensure financial accountability were perceived to be robust, with concerns about accountability only raised when finance came from outside the government system, for example, from specific donors. District level respondents were unhappy about the seeming lack of accountability of the medication procurement agency for reportedly poor performance, but also spoke of the problems with accountability, for instance in terms of quality of medication, when they were forced to purchase medication from the private sector.

Respondents expressed less confidence in the transparency of decisions. Most respondents did not perceive that they could easily access information about budgets and planning decisions in the public domain. Other respondents countered that the difficulty was more one of collating the information rather than a problem of transparency. Questions were also raised about the transparency of systems for employing administrative and health facility heads.

\section{Discussion}

In this qualitative study of key informants from Ethiopia, strengths and weaknesses of health system governance to support mental health care scale-up were identified through systematic application of an evaluation framework. Many of the governance challenges facing Ethiopia have been identified previously as barriers across LMICs in reports based on expert consensus [3]. A cross-country analysis of health system governance for the Emerald countries (Ethiopia, India, Nepal, Nigeria, South Africa, Uganda) has drawn out commonalities in challenges and opportunities [39]. Our study extends this previous work by identifying the areas of relative strength and weakness specific to the Ethiopian context which may usefully inform the ongoing efforts to scale-up mental health care.

The high level governmental commitment, development of a National Mental Health Strategy, expansion of specialist mental health workers, integration of mental health into health extension worker upgrade training, specification of targets in the health care plan and dedicated budget are indicative of strong strategic vision for mental health care scale-up in Ethiopia. These aspects of HSG have been found to be weak in evaluations of HSGs in other LMICs [9], including other Emerald study sites [32]. Ethiopia has benefited from a strong tradition of population level mental health research which has served to quantify the extent and burden of mental disorders within Ethiopia and strengthen arguments underpinning advocacy efforts [18]. Alongside this, the Toronto-Addis Ababa Psychiatry Project (TAAPP) has contributed to the expansion of psychiatrists within Ethiopia through support of an in-country training programme [40]. TAAPP focuses on broad training competencies, including the 'psychiatrist as advocate', which has helped to build up a critical mass of informed mental health professionals ready to engage with the Federal Ministry of Health. These factors, combined with committed and visionary leadership within the Ministry, mean that Ethiopia is in a substantially better position than 10 years ago to expand mental health care to the population.

Nonetheless, it was clear from this study that high level government commitment and a conducive policy context may not, by themselves, provide sufficient governance for successful scale-up. A recurring theme from respondents in our study was the need to enhance awareness, leadership, motivation and expertise in mental health care planning in order to successfully expand mental health care at the regional, zonal and district health administration levels. The current approach to scale-up of mental health care in Ethiopia involves working with the Regional Health Bureaus to train health workers from selected primary healthcare facilities across the region, thus bypassing the district-led planning system. With this approach, newly trained health workers return to their facilities but do not have system level support for delivery of mental healthcare. Such standalone mental health training interventions for primary care workers often have minimal 
impact on improving healthcare [41]. In the PRIME study in Ethiopia, brief training of primary care workers is being combined with engagement of the district health administration [29]. An initial half-day workshop to raise awareness about mental health led to the assignment of a mental health co-ordinator within the district health office. The PRIME team has provided ongoing technical support to the mental health co-ordinator with respect to mental health care planning, including medication procurement and training of trainers and supervisors for mental health care. The need to develop local leadership for mental health care accords with findings from the field of reproductive health, where managerial and leadership competence were identified as key system level factors distinguishing successful from nonsuccessful implementation in primary care in rural Ethiopia [5]. In a recent systematic review of capacity-building initiatives for policy-makers and planners to strengthen mental health systems in LMICs [42], some promising approaches were identified. Although evaluation of the impact of such capacity-building approaches was limited, important ingredients for success appeared to include raising awareness of the public health relevance of mental health and tackling stigma, providing technical support within the context of an ongoing mentoring relationship and establishing networks for support and experiencesharing. Programmes to equip mental health specialists with the leadership skills needed to support system reform are also needed [43, 44]. In Ethiopia, there is a need to equip all cadres of mental health specialist with competencies in leadership and service planning to complement clinical skills.

The need for better co-ordination and involvement of stakeholders to allow for participatory planning was an important theme. At present there are limited mechanisms in Ethiopia for stakeholders to get involved in mental health care development and to have their voices heard. Low levels of mobilisation of service users within the country contributes to the problem and marks Ethiopia out compared to the other Emerald countries [32-34]. There are examples of successful involvement of service users in other LMICs in mental health system strengthening activities, including planning, service monitoring and advocacy, although the lack of rigorous evaluation of the impact of such initiatives is a barrier to more widespread uptake [45]. The Emerald project is piloting and evaluating approaches to encouraging more grassroots involvement of mental health service users to strengthen governance of integrated mental health care scale-up [46]. The importance of identifying leverage points within complex systems has emerged from analysis of successful mental health implementation projects in selected middle-income countries [8]. Enhanced service user involvement could be an important leverage point in the Ethiopian mental health system, with the potential to address a number of the main concerns arising in our analysis of governance: stigma, low awareness and demand, equitable access to care, quality of care, transparency and accountability of services.

Mental health integrated into primary care was perceived by all respondents to be more responsive and efficient than the existing centralised system of specialist mental health care. However, there was concern about how integrated care could be achieved in practice unless critical system level barriers were addressed. Chief among the barriers were low demand for mental health care (due to low awareness and stigma), lack of affordability of long-term care and inadequate supervision from mental health specialists. These factors may result in low uptake of care, expiry of medications (compounding supply chain obstacles), ineffectiveness of care and, ultimately, a lack of sustainability of services. Raising awareness about mental health conditions and their treatability in the community, combined with training community members to detect and refer possible cases, has been used successfully to increase uptake of mental health care in the PRIME implementation district [29]. This is a potentially scalable approach which could be integrated into health extension worker activities in order to support the national programme of mental health care scale-up. Given the brief nature of mhGAP training for primary care workers, ongoing supervision, mentoring and refresher training is essential for quality of care and to give primary care workers the confidence and impetus to deliver mental health care [3]. The shortage of mental health specialists in Ethiopia and their orientation towards hospital-based delivery of clinical care in hospitals means that regular supervision of newly trained primary care workers is not currently achievable. There is a need to expand the remit of mental health specialists to include supervision of primary care workers and to ensure that pre-service training equips specialists with the requisite skills. In the meantime, training the existing pool of non-specialist health worker supervisors in each district to also cover mental health care and combining this with telephone consultation with mental health specialists could help to address this important gap.

The inadequacy of existing mechanisms for the routine monitoring and evaluation of mental health care scale-up was considered to undermine several aspects of health system governance, including the responsiveness, effectiveness, efficiency, quality and equity of care. At present the Ethiopian health management information system (HMIS) only captures data about service utilisation on people with a 'mental or behavioural disorder' and 'epilepsy' which provides limited information to evaluate the 
service and support planning [47]. The optimal HMIS indicators required to monitor integrated mental health care in LMICs are not known. In a recent Delphi consensus exercise with experts from across six LMICs, a combination of indicators were endorsed to allow measurement of effective coverage (defined as "the proportion of people in need of a service who gain the intended health benefit from that service" [48]): items monitoring need for services, utilisation of care for specific mental health conditions, quality of care and financial protection [49]. An evaluation of the validity and utility of these selected indicators is underway in the Emerald countries.

\section{Strengths and limitations}

Strengths of our study include the systematic and theoretically-driven approach to exploration of health system governance challenges. We included a range of relevant stakeholders, both at the national/regional level and the district level, thus encompassing perspectives from frontline managers as well as high level policy makers. The validity of our findings was enhanced by the opportunity to present our preliminary findings at the $\mathrm{FMOH}$ consultation meeting on mental health care scale-up. The study was limited by the relatively small number of interviews at all levels which reflected the limited pool of planners and managers with relevant expertise. As a consequence, theoretical saturation may not have been achieved. Service users and caregivers were not included in the current analysis because a separate study was conducted to investigate their involvement in aspects of mental health system strengthening, including governance [50].

\section{Conclusions}

In order to support the scale-up of mental health care in Ethiopia, there is a critical need to strengthen leadership, co-ordination and capacity to plan mental health care at the national, regional, zonal and district levels, mobilise service users for greater involvement, expand monitoring and evaluation systems to capture mental health information and address widespread stigma and low awareness.

\section{Additional files}

Additional file 1. Topic guides.

Additional file 2. Adapted analysis framework for health system governance in Ethiopia.

Additional file 3. Supporting quotations for each theme.

Additional file 4. Analysis matrix 1.

Additional file 5. Analysis matrix 2.

\section{Abbreviations}

AFFIRM: Africa Focus on Intervention Research for Mental Health; Emerald: emerging mental health systems in low- and middle-income countries; HIV/
AIDS: human immunodeficiency virus/acquired immunodeficiency syndrome; HSG: health system governance; LMICs: low- and middle-income countries; mhGAP: mental health Gap Action Programme; NCDs: non-communicable diseases; PHC: primary health care; PRIME: Programme for Improving Mental health carE; TAAPP: Toronto-Addis Ababa Psychiatry Programme; TB: tuberculosis; WHO: World Health Organisation.

\section{Authors' contributions}

$\mathrm{CH}, \mathrm{AA}, \mathrm{DLM}, \mathrm{IP}$ and FK designed the study. TE and DA collected the data. $\mathrm{CH}$, TE and DA led the analysis, with input from AA and AF. CH wrote the first draft of the manuscript. CH, AA, GT, IP, FK, DLM, MS, TE, DA and AF contributed to the interpretation of the findings and commented on the drafted manuscript. All authors read and approved the final manuscript.

\section{Author details}

${ }^{1}$ Department of Psychiatry, School of Medicine, College of Health Sciences, Addis Ababa University, Addis Ababa, Ethiopia. ${ }^{2}$ Centre for Global Mental Health, Health Services and Population Research Department, Institute of Psychiatry, Psychology and Neuroscience, King's College London, London, UK. ${ }^{3}$ Department of Psychological Medicine, Centre for Affective Disorders, Institute of Psychiatry, Psychology and Neuroscience, King's College London, London, UK. ${ }^{4}$ Butabika National Referral and Teaching Hospital, Makerere University, Kampala, Uganda. ${ }^{5}$ Research Development \& Support Division, Faculty of Medicine \& Health Sciences, Stellenbosch University, Cape Town, South Africa. ${ }^{6}$ School of Nursing and Public Health, University of KwaZuluNatal, Durban, South Africa. ${ }^{7}$ School of Applied Social Sciences, University of KwaZulu-Natal, Durban, South Africa.

\section{Acknowledgements}

We are grateful to all of the participants for giving their time and participating actively.

The partner organizations involved in the Emerald program are Addis Ababa University (AAU), Ethiopia; Butabika National Mental Hospital (BNH), Uganda; GABO:mi Gesellschaft für Ablauforganisation:milliarium GmBH \& Co KG (GABO:mi), Germany; HealthNet TPO, Netherlands; King's College London (KCL), United Kingdom (UK); Public Health Foundation of India (PHFI), India; Transcultural Psychosocial Organization Nepal (TPO Nepal), Nepal; Universidad Autonoma de Madrid (UAM), Spain; University of Cape Town (UCT), South Africa; University of Ibadan (UI), Nigeria; University of KwaZulu-Natal (UKZN), South Africa; and World Health Organization (WHO), Switzerland.

The Emerald program is led by Prof. Graham Thornicroft at KCL. The project coordination group consists of Prof. Atalay Alem (AAU), Prof. José Luis Ayuso-Mateos (UAM), Dr. Dan Chisholm (WHO), Dr. Stefanie Fülöp (GABO:mi), Prof. Oye Gureje (UI), Dr. Charlotte Hanlon (AAU), Dr. Mark Jordans (HealthNet TPO; TPO Nepal; KCL), Dr. Fred Kigozi (BNH), Prof. Crick Lund (UCT), Prof. Inge Petersen (UKZN), Dr. Rahul Shidhaye (PHFI), and Prof. Graham Thornicroft (KCL).

Parts of the program are also coordinated by Ms. Shalini Ahuja (PHFI), Dr. Jibril Omuya Abdulmalik (UI), Ms. Kelly Davies (KCL), Ms. Sumaiyah Docrat (UCT), Dr. Catherine Egbe (UKZN), Dr. Sara Evans-Lacko (KCL), Dr. Margaret Heslin (KCL), Dr. Dorothy Kizza (BNH), Ms. Lola Kola (UI), Dr. Heidi Lempp (KCL), Dr. Pilar López (UAM), Ms. Debra Marais (UKZN), Ms. Blanca Mellor (UAM), Mr. Durgadas Menon (PHFI), Dr. James Mugisha (BNH), Ms. Sharmishtha Nanda (PHFI), Dr. Anita Patel (KCL), Ms. Shoba Raja (BasicNeeds, India; KCL), Dr. Maya Semrau (KCL), Mr. Joshua Ssebunya (BNH), Mr. Yomi Taiwo (UI), and Mr. Nawaraj Upadhaya (TPO Nepal).

The Emerald program's scientific advisory board includes A/Prof. Susan Cleary (UCT), Prof. Derege Kebede (WHO, Regional Office for Africa), Prof. Harry Minas (University of Melbourne, Australia), Mr. Patrick Onyango (TPO Uganda), Prof. Jose Luis Salvador Carulla (University of Sydney, Australia), and Dr. R. Thara (Schizophrenia Research Foundation (SCARF), India).

The following individuals are members of the Emerald consortium: Dr. Kazeem Adebayo (UI), Ms. Jennifer Agha (KCL), Ms. Ainali Aikaterini (WHO), Dr. Gunilla Backman (London School of Hygiene and Tropical Medicine; KCL), Mr. Piet Barnard (UCT), Dr. Harriet Birabwa (BNH), Ms. Erica Breuer (UCT), Mr. Shveta Budhraja (PHFI), Amit Chaturvedi (PHFI), Mr. Daniel Chekol (AAU), Mr. Naadir Daniels (UCT), Mr. Bishwa Dunghana (TPO Nepal), Ms. Gillian Hanslo (UCT), Ms. Edith Kasinga (UCT), Ms. Tasneem Kathree (UKZN), Mr. Suraj Koirala (TPO Nepal), Prof. Ivan Komproe (HealthNet TPO), Dr. Mirja Koschorke (KCL), Mr. Domenico Lalli (European Commission), Mr. Nagendra Luitel (TPO Nepal), Dr. David McDaid (KCL), Ms. Immaculate Nantongo (BNH), Dr. Sheila Ndyanabangi (BNH), Dr. Bibilola Oladeji (UI), Prof. Vikram Patel (KCL), Ms. Louise Pratt 
(KCL), Prof. Martin Prince (KCL), Ms. M Miret (UAM), Ms. Warda Sablay (UCT), Mr. Bunmi Salako (UI), Dr. Tatiana Taylor Salisbury (KCL), Dr. Shekhar Saxena (WHO), Ms. One Selohilwe (UKZN), Dr. Ursula Stangel (GABO:mi), Prof. Mark Tomlinson (UCT), Dr. Abebaw Fekadu (AAU), and Ms. Elaine Webb (KCL).

\section{Competing interests}

GT is supported by the National Institute for Health Research (NIHR) Collaboration for Leadership in Applied Health Research and Care South London at King's College London Foundation Trust. The views expressed are those of the author(s) and not necessarily those of the NHS, the NIHR or the Department of Health. GT acknowledges financial support from the Department of Health via the National Institute for Health Research (NIHR) Biomedical Research Centre and Dementia Unit awarded to South London and Maudsley NHS Foundation Trust in partnership with King's College London and King's College Hospital NHS Foundation Trust. GT and MS are supported by the European Union Seventh Framework Programme (FP7/2007-2013) Emerald project.

\section{Availability of data and materials}

Supporting data for the themes are included in Additional file 4. The data may be requested from the corresponding author for verification of the analyses in this paper.

\section{Ethics approval and consent to participate}

Ethical approval was obtained from the Institutional Review Board of the College of Health Sciences, Addis Ababa University (Reference No. 074/13/Psy). Participation was voluntary and only took place after informed consent had been given.

\section{Funding}

The research leading to these results is funded by the European Union's Seventh Framework Programme (FP7/2007-2013) under Grant Agreement No. 305968.

\section{Publisher's Note}

Springer Nature remains neutral with regard to jurisdictional claims in published maps and institutional affiliations.

\section{Received: 23 February 2017 Accepted: 24 May 2017} Published online: 08 June 2017

\section{References}

1. World Health Organization. Mental Health Gap Action Programme (mhGAP): scaling up care for mental, neurological, and substance use disorders. Geneva: WHO; 2008.

2. Eaton J, McCay L, Semrau M, Chatterjee S, Baingana F, Araya R, Ntulo C, Thornicroft G, Saxena S. Scale up of services for mental health in lowincome and middle-income countries. Lancet. 2011;378(9802):1592-603.

3. Thornicroft G, Alem A, Santos RA, Barley E, Drake RE, Gregorio G, Hanlon C, Ito H, Latimer E, Law A, et al. WPA guidelines on steps, obstacles and mistakes to avoid in the implementation of community mental health care. World Psychiatry. 2010;9(2):67-77.

4. Saraceno B, van Ommeren M, Batniji R, et al. Barriers to improvement of mental health services in low-income and middle-income countries. Lancet. 2007;370:1174.

5. Bradley EH, Byam P, Alpern R, Thompson JW, Zerihun A, Abebe Y, Curry LA. A systems approach to improving rural care in Ethiopia. (Erratum appears in PLoS ONE. 2012 May;7(5): doi:10.1371/annotation/7faaa94384f2-48e9-9c7e-e19b5b037716. Note: Abeb, Yigeremu [corrected to Abebe, Yigeremu]). PLoS ONE. 2012;7(4):e35042.

6. World Health Organization. Everybody's business: strengthening health systems to improve health outcomes: WHO's framework for action. Geneva: WHO; 2007.

7. de Savigny D, Adam T. Systems thinking for health system strengthening. In: Alliance for health policy and systems research. Geneva: WHO; 2009.

8. Kidd SA, Madan A, Rallabandi S, Cole DC, Muskat E, Raja S, Wiljer D, Aylward D, McKenzie K. A multiple case study of mental health interventions in middle income countries: considering the science of delivery. PLoS ONE. 2016;11(3):e0152083.

9. Mikkelsen-Lopez I, Wyss K, de Savigny D. An approach to addressing governance from a health system framework perspective. BMC Int Health Hum Rights. 2011;11:13.

10. Projected population for 2015. http://www.csa.gov.et/. Accessed 7 June 2017.

11. Federal Democratic Republic of Ethiopia Ministry of Health. National mental health strategy, 2012/13-2015/16. Addis Ababa: Federal Ministry of Health; 2012.

12. Alem A, Kebede D, Fekadu A, Shibre T, Fekadu D, Beyero T, Medhin G, Negash A, Kullgren G. Clinical course and outcome of schizophrenia in a predominantly treatment-naive cohort in rural Ethiopia. Schizophr Bull. 2009;35(3):646-54.

13. Fekadu A, Kebede D, Alem A, Fekadu D, Mogga S, Negash A, Medhin G, Beyero T, Shibre T. Clinical outcome in bipolar disorder in a communitybased follow-up study in Butajira, Ethiopia. Acta Psychiatr Scand. 2006;114(6):426-34

14. Fekadu A, Medhin G, Kebede D, Alem A, Cleare A, Prince M, Hanlon C, Shibre T. Excess mortality in severe mental disorders: a 10-year populationbased cohort study in rural Ethiopia. Br J Psychiatry. 2014;206(4):289-96.

15. Shibre T, Medhin G, Alem A, Kebede D, Teferra S, Jacobsson L, Kullgren G, Hanlon C, Fekadu A. Long-term clinical course and outcome of schizophrenia in rural Ethiopia: 10-year follow-up of a population-based cohort. Schizophr Res. 2014;161(2-3):414-20.

16. Zergaw A, Hailemariam D, Alem A, Kebede D. A longitudinal comparative analysis of economic and family caregiver burden due to bipolar disorder. Afr J Psychiatry. 2008;11:191-8.

17. Shibre T, Negash A, Kullgren G, Kebede D, Alem A, Fekadu A, Fekadu D, Madhin $G$, Jacobsson L. Perception of stigma among family members of individuals with schizophrenia and major affective disorders in rural Ethiopia. Soc Psychiatry Psychiatr Epidemiol. 2001;36(6):299-303.

18. Fekadu A, Thornicroft G. Global mental health: perspectives from Ethiopia. Glob Health Action. 2014;7:25447. doi:10.3402/gha.v7.25447.

19. Federal Democratic Republic of Ethiopia Ministry of Health. Health sector transformation plan: 2015/2016-2019/2020. Addis Ababa: FMOH; 2015.

20. Federal Ministry of Health. Health policy of the transitional government of Ethiopia. Addis Ababa: Federal Ministry of Health; 1993.

21. Federal Democratic Republic of Ethiopia Ministry of Health. National mental health strategy, 2012/13-2015/16. Addis Ababa: Ministry of Health; 2012.

22. World Health Organization. Mental health atlas 2014. Geneva: WHO; 2014

23. United Nations. United Nations General Assembly A/61/611 convention on the rights of persons with disabilities Dec 2006. 2006. https://www. un.org/development/desa/disabilities/convention-on-the-rights-ofpersons-with-disabilities.html. Accessed 19 Sep 2016.

24. Ethiopian Health Insurance Agency. Evaluation of community-based health insurance pilot schemes in Ethiopia: final report. Addis Ababa: Ethiopian Health Insurance Agency; 2015.

25. Federal Ministry of Health of Ethiopia, World Health Organization. mhGAP in Ethiopia: proof of concept 2013. Geneva: WHO; 2013.

26. World Health Organization. Mental Health Gap Action Programme Intervention Guide (mhGAP-IG) for mental, neurological and substance use disorders in non-specialized health settings, version 2.0. Geneva: WHO; 2016.

27. Federal Democratic Republic of Ethiopia Ministry of Health. Non-communicable diseases, emergency care and mental health part 2 mental health problems. Blended learning module for the health extension programme. Addis Ababa: Federal Democratic Republic of Ethiopia Ministry of Health Ethiopia; 2011. http://www.open.edu/openlearnworks/course/ view.php?id=19. Accessed 15 Sep 2016.

28. Hanlon C, Luitel NP, Kathree T, Murhar V, Shrivasta S, Medhin G, Ssebunnya J, Fekadu A, Shidhaye R, Petersen I, et al. Challenges and opportunities for implementing integrated mental health care: a district level situation analysis from five low- and middle-income countries. PLOS ONE. 2014;9(2):e88437.

29. Fekadu A, Hanlon C, Medhin G, Alem A, Selamu M, Giorgis T, Shibre T, Teferra S, Tegegn T, Breuer E, et al. Development of a scalable mental healthcare plan for a rural district in Ethiopia. Br J Psychiatry Suppl. 2015. doi:10.1192/bjp.bp.1114.153676. 
30. Hanlon C, Alem A, Medhin G, Shibre T, Ejigu DA, Negussie H, Dewey M, Wissow L, Prince M, Susser E, et al. Task sharing for the care of severe mental disorders in a low-income country (TaSCS): study protocol for a randomised, controlled, non-inferiority trial. Trials. 2016;17:76. doi:10.1186/s13063-13016-11191-x.

31. Semrau M, Evans-Lacko S, Alem A, Ayuso-Mateos JL, Chisholm D, Gureje O, Hanlon C, Jordans M, Kigozi F, Lempp H, et al. Strengthening mental health systems in low- and middle-income countries: the Emerald programme. BMC Med. 2015;13:79.

32. Abdulmalik J, Kola L, Gureje O. Mental health system governance in Nigeria: challenges, opportunities and strategies for improvement. Glob Ment Health. 2016;3:e9. doi:10.1017/gmh.2016.1012.

33. Marais DL, Petersen I. Health system governance to support integrated mental health care in South Africa: challenges and opportunities. Int J Ment Health Syst. 2015;9:14

34. Mugisha J, Ssebunnya J, Kigozi F. Towards understanding governance issues in integration of mental health into primary health care in Uganda. Int J Ment Health Syst. 2016;10:25. doi:10.1186/ s13033-13016-10057-13037.

35. Siddiqi S, Masud TI, Nishtar S, Peters DH, Sabri B, Bile KM, Jama MA Framework for assessing governance of the health system in developing countries: gateway to good governance. Health Policy. 2009;90(1):13-25.

36. Programme UND. Human development report 2015. Work for human development. New York: UNDP; 2015.

37. Gale NK, Heath G, Cameron E, Rashid S, Redwood S. Using the framework method for the analysis of qualitative data in multi-disciplinary health research. BMC Med Res Methodol. 2013;13:117.

38. ICT Services and System Development and Division of Epidemiology and Global Health. OpenCode 4.0. Umeå: University of Umeå; 2013. http:// www.phmed.umu.se/english/units/epidemiology/research/open-code/. Accessed 15 Sep 2016.

39. Petersen I, Marais D, Abdulmalik J, Ahuja S, Alem A, Chisholm D, Egbe C, Gureje $\mathrm{O}$, Hanlon C, Lund C, et al. Strengthening mental health system governance in six low- and middle-income countries in Africa and South Asia: challenges, needs and potential strategies. Health Policy Plan. 2017. doi:10.1093/heapol/czx1014.

40. Alem A, Pain C, Araya M, Hodges BD. Co-creating a psychiatric resident program with Ethiopians, for Ethiopians, in Ethiopia: the Toronto Addis Ababa Psychiatry Project (TAAPP). Acad Psychiatry. 2010;34(6):424-32.

41. Gilbody SM, Whitty PM, Grimshaw JM, Thomas RE. Improving the detection and management of depression in primary care. Qual Saf Health Care. 2003;12(2):149-55.
42. Keynejad R, Semrau M, Toynbee M, Evans-Lacko S, Lund C, Gureje O, Ndyanabangi S, Courtin E, Abdulmalik JO, Alem A, Fekadu A, Thornicroft $\mathrm{G}$, Hanlon C. Building the capacity of policy-makers and planners to strengthen mental health systems in low- and middle-income countries: a systematic review. BMC Health Serv Res. 2016;16(1):601.

43. Abdulmalik J, Fadahunsi W, Kola L, Nwefoh E, Minas H, Eaton J, Gureje O. The Mental Health Leadership and Advocacy Program (mhLAP): a pioneering response to the neglect of mental health in Anglophone West Africa. Int J Ment Health Syst. 2014;8:5. doi:10.1186/1752-4458-1188-1185.

44. Minas $\mathrm{H}$. The centre for international mental health approach to mental health system development. Harv Rev Psychiatry. 2012;20:37-47.

45. Semrau M, Lempp H, Keynejad R, Evans-Lacko S, Mugisha J, Raja S, Lamichhane J, Alem A, Thornicroft G, Hanlon C. Service user and caregiver involvement in mental health system strengthening in low- and middle-income countries: systematic review. BMC Health Serv Res. 2016. doi:10.1186/s12913-12016-11323-12918.

46. Samudre SD, Shidhaye RR, Ahuja S, Nanda S, Khan A, Evans-Lacko S, Hanlon C. Service user involvement for mental health system strengthening in India: a qualitative study. BMC Psychiatry. 2016;16:269. doi:10.1186/ s12888-12016-10981-12888.

47. Upadhaya N, Jordans MJD, Abdulmalik J, Ahuja S, Alem A, Hanlon C, Kigozi F, Kizza D, Lund C, Semrau M, Shidhaye R, Thornicroft G, Komproe $I H$, Gureje O. Information systems for mental health in six low and middle income countries: cross country situation analysis. Int J Ment Health Syst. 2016;10:60.

48. De Silva M, Lee L, Fuhr D, Rathod S, Chisholm D, Schellenberg J, Patel $\checkmark$. Estimating the coverage of mental health programmes: a systematic review. Int J Epidemiol. 2014;43:341-53.

49. Jordans MJD, Chisholm D, Semrau M, Upadhaya N, Abdulmalik J, Ahuja S, Alem A, Hanlon C, Kigozi F, Mugisha J, et al. Indicators for routine monitoring of effective mental healthcare coverage in low- and middle-income settings: a Delphi study. Health Policy Plan. 2016;1-7. doi:10.1093/heapol/czw1040.

50. Abayneh S, Lempp H, Alem A, Alemayehu D, Eshetu T, Lund C, Semrau M, Thornicroft G, Hanlon C. Service user involvement in mental health system strengthening in a rural African setting: qualitative study. BMC Psychiatry. 2017;17:187. doi:10.1186/s12888-017-1352-9.

\section{Submit your next manuscript to BioMed Central and we will help you at every step:}

- We accept pre-submission inquiries

- Our selector tool helps you to find the most relevant journal

- We provide round the clock customer support

- Convenient online submission

- Thorough peer review

- Inclusion in PubMed and all major indexing services

- Maximum visibility for your research

Submit your manuscript at www.biomedcentral.com/submit
O Biomed Central 